\title{
$\mathcal{P}$ T-Symmetric Phonon Laser
}

\author{
H. Jing, ${ }^{1,2}$ S. K. Özdemir, ${ }^{1,3}$ Xin-You Lü, ${ }^{1}$ Jing Zhang, ${ }^{1,4}$ Lan Yang, ${ }^{3}$ and Franco Nori ${ }^{1,5}$ \\ ${ }^{1}$ CEMS, RIKEN, Saitama, 351-0198, Japan \\ ${ }^{2}$ Department of Physics, Henan Normal University, Xinxiang 453007, P.R. China \\ ${ }^{3}$ Electrical and Systems Engineering, Washington University, St. Louis, Missouri 63130, USA \\ ${ }^{4}$ Department of Automation, Tsinghua University, Beijing 100084, P.R. China \\ ${ }^{5}$ Physics Department, University of Michigan, Ann Arbor, MI 48109-1040, USA
}

(Dated: August 4, 2014)

\begin{abstract}
By exploiting recent developments associated with coupled microcavities, we introduce the concept of $\mathcal{P} \mathcal{T}$-symmetric phonon laser with balanced gain and loss. This is accomplished by introducing gain to one of the microcavities such that it balances the passive loss of the other. In the vicinity of the gain-loss balance, a strong nonlinear relation emerges between the intracavity photon intensity and the input power. This then leads to a giant enhancement of both optical pressure and mechanical gain, resulting in a highly efficient phonon-lasing action. These results provide a promising approach for manipulating optomechanical systems through $\mathcal{P} \mathcal{T}$-symmetric concepts. Potential applications range from enhancing mechanical cooling to designing phonon-laser amplifiers.
\end{abstract}

PACS numbers: 42.50.-p, 03.75.Pp, 03.70.+k

Recent advances in materials science and nanofabrication have led to spectacular achievements in cooling classical mechanical objects into the subtle quantum regime (e.g., [1-4] ). These results are having a profound impact on a wide range of research topics, from probing basic rules of classical-to-quantum transitions [4 7] to creating novel devices operating in the quantum regime, e.g. ultra-weak force sensors [8] or electric-to-optical wave transducers [9, 10]. The emerging field of cavity optomechanics (COM) [1] is also experiencing rapid evolution that is driven by studies aimed at understanding the underlying physics and by the fabrication of novel structures and devices enabled by recent developments in nanotechnology.

The basic COM system includes a single resonator, where a highly-efficient energy transfer between the mechanical mode and intracavity photons is enabled by detuning an input laser from the cavity resonance [1]. A new extension, closely related to the present study, is the photonic molecule or compound microresonators [11 13], where a tunable optical tunneling can be exploited to bypass the frequency detuning requirement [12]. More strikingly, in this architecture, an analogue of two-level optical laser is provided by phonon-mediated transitions between two optical supermodes [13]. This phonon laser [13, 14] provides the core technology to integrate coherent phonon sources, detectors, and waveguides - allowing the study of nonlinear phononics 15] and the operation of functional phononic devices [16].

In parallel to these works, intense interest has also emerged recently in $\mathcal{P} \mathcal{T}$-symmetric optics [17 19]. A variety of optical structures, whose behaviors can be described by parity-time $(\mathcal{P} \mathcal{T})$ symmetric Hamiltonians, have been fabricated [17]. These exotic structures provide unconventional and previously-unattainable control of light [1, 18, 19, 21]. In very recent work, by manipu- lating the gain (in one active or externally-pumped resonator) to loss (in the other, passive, one) ratio, Ref. [1] realized an optical compound structure with remarkable $\mathcal{P} \mathcal{T}$-symmetric features, e.g. field localization in the active resonator and accompanied enhancement of optical nonlinearity leading to nonreciprocal light transmission. However, COM properties underlying the phonon-laser action in the $\mathcal{P} \mathcal{T}$-symmetric regime, where gain and loss are balanced, remain largely unexplored.

Here we study a $\mathcal{P} \mathcal{T}$-symmetric COM system which is formed by two coupled microcavities, one of which has passive loss (passive resonator $\mathbb{R}_{\gamma}$ : no optical gain) and the other has optical gain (active resonator $\mathbb{R}_{\kappa}$ ) balancing the loss of $\mathbb{R}_{\gamma}$. In contrast to passive COM, with single or coupled passive resonators, $\mathcal{P} \mathcal{T}$-symmetric COM features a transition from linear to nonlinear regimes for intracavity photon intensity, by controlling the gain-loss ratio. In this nonlinear regime, a giant enhancement of both optical pressures and mechanical gain can be realized. Consequently, in the $\mathcal{P} \mathcal{T}$-symmetric regime, an ultralowthreshold phonon laser is achievable by approaching the gain-loss balance. We note that the phonon lasing action, through energy exchange of two nondegenerate optical supermodes, exists only in the $\mathcal{P} \mathcal{T}$-symmetric regime. The enhanced nonlinearity is responsible for the ultralow threshold of the phonon laser and can also be useful for studying a wide variety of optomechanical processes, e.g., single-photon COM [23] or phononic mixing [16, 24]. All relevant parameters and techniques are well within the reach of current experimental capabilities.

We consider two coupled whispering-gallery-mode (WGM) microtoroid resonators. One of the microtoroids is fabricated from silica and has passive loss (passive resonator $\mathbb{R}_{\gamma}$ ), whereas the other microtoroid is fabricated from silica doped with $\mathrm{Er}^{3+}$ ions (active resonator $\mathbb{R}_{\kappa}$ ). $\mathrm{Er}^{3+}$ ions emit photons in the $1550 \mathrm{~nm}$ band when $\mathbb{R}_{\kappa}$ 
is optically pumped with a light in the $1460 \mathrm{~nm}$ band. This provides the optical gain $\kappa$ to compensate for the optical losses and to amplify weak signal light in the $1550 \mathrm{~nm}$ band [1]. Evanescent coupling between the two resonators exists only in the $1550 \mathrm{~nm}$ band, assuring that the light in the $1460 \mathrm{~nm}$ band only resides in $\mathbb{R}_{\kappa}[1]$. The amplified light in the $1550 \mathrm{~nm}$ band then serves as the pump for a mechanical mode (frequency $\omega_{m}$ and effective mass $m$ ) contained in the passive resonator $\mathbb{R}_{\gamma}[13]$. In order to couple external light into and out of the WGMs, each microtoroid is coupled to a different tapered-fiber waveguide (see Fig. 1).

By achieving population inversion in this system, the stimulated emission of phonons can occur, in close analogy to an optical laser. A phonon laser operating with threshold power $\sim 7 \mu \mathrm{W}$ has been already demonstrated [13] with two passive resonators. Extending this system to involve gain and loss yields the Heisenberg equations of motion $(\hbar=1)$

$$
\begin{aligned}
& \dot{a}_{1}=\kappa a_{1}+i J a_{2}+\sqrt{2 \kappa} a^{\mathrm{in}}, \\
& \dot{a}_{2}=-\gamma a_{2}+i J a_{1}+i g a_{2} x, \\
& \ddot{x}+\Gamma_{m} \dot{x}+\omega_{m}^{2} x=\frac{g}{m} a_{2}^{\dagger} a_{2}+\frac{\varepsilon^{\text {in }}}{m},
\end{aligned}
$$

where $x=x_{0}\left(b+b^{\dagger}\right)$ is the mechanical position operator with $x_{0}=\left(2 m \omega_{m}\right)^{-1 / 2}$ and the operator $b$ denoting the phonon mode, $g=\omega_{c} / R$ is the COM coupling coefficient, $J$ is the inter-cavity coupling rate, $R$ is the microtoroid radius, and $\gamma=\omega_{c} / Q_{c}$ is the optical loss. The underlying resonances are assumed degenerate with frequency $\omega_{c}=c / \lambda$, while $a_{1,2}$ and $b$ denote the lowering operators for the optical and mechanical modes, respectively. $\Gamma_{m}$ is the mechanical damping rate and the operator $\varepsilon^{\text {in }}$ describes the thermal Brownian noise resulting from the coupling of the resonators to the environment. The operator $a^{\text {in }}$ is the optical noise operator describing the signal light incident on $\mathbb{R}_{\kappa}$; its mean value $f_{\text {in }}=\left\langle a^{\text {in }}(t)\right\rangle$ is positive, and its fluctuations denoted by $\delta a^{\mathrm{in}}(t)$ are assumed to be delta-correlated [19], adding vacuum noise to the resonator modes

$$
\left\langle\delta a^{\mathrm{in}, \dagger}(t) \delta a^{\mathrm{in}}\left(t^{\prime}\right)\right\rangle=\delta\left(t-t^{\prime}\right), \quad\left\langle\delta a^{\mathrm{in}}(t) \delta a^{\mathrm{in}, \dagger}\left(t^{\prime}\right)\right\rangle=0 .
$$

By setting the time derivatives in Eqs.(11)-(3) to zero, we find the steady state of the dynamical variables as

$$
\begin{aligned}
a_{1, s} & =\frac{-\sqrt{2 \kappa} f_{\text {in }}}{\kappa-J^{2} /\left(\gamma-i g x_{s}\right)}, \\
a_{2, s} & =\frac{-i J \sqrt{2 \kappa} f_{\text {in }}}{\kappa \gamma-J^{2}-i \kappa g x_{s}}, \quad x_{s}=\frac{g\left|a_{2, s}\right|^{2}}{m \omega_{m}^{2}} .
\end{aligned}
$$

The expressions of $a_{2, s}$ and $x_{s}$ can be combined to give

$$
\frac{2 \kappa J^{2} P_{\text {in }} / R}{\left(\kappa \gamma-J^{2}\right)^{2}+\left(\kappa g x_{s}\right)^{2}}=m \omega_{m}^{2} x_{s}
$$

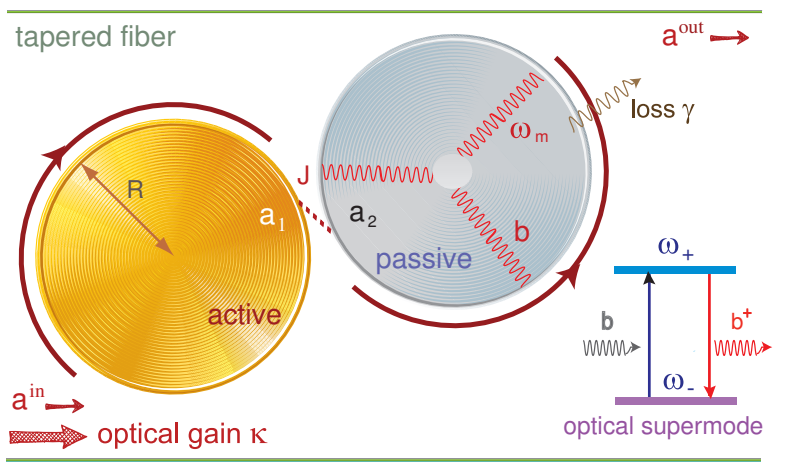

FIG. 1: (Color online) Gain-enhanced optomechanics in compound whispering-gallery resonators. The optical tunnelling rate $J$ is tuned by changing the distance between $\mathbb{R}_{\kappa}$ and $\mathbb{R}_{\gamma}$. The corresponding optical supermodes coupled by phonons are also plotted.

which shows the balance of the radiation and spring forces. Here we have used $P_{\text {in }}=\omega_{c}\left|f_{\text {in }}\right|^{2}$, with $P_{\text {in }}$ denoting the power of the signal light incident on $\mathbb{R}_{\kappa}$. The cubic equation about $x_{s}$ characterizes the occurrence of bistability at higher input power $P_{\text {in }}$, as in passive COM systems [4, 5].

If the coupling rate, gain and loss satisfy the condition $J^{2}=\kappa \gamma$, there exists only one solution that is always positive

$$
a_{2, s}=\frac{\sqrt{2 \gamma} f_{\mathrm{in}}}{g x_{s}}, \quad x_{s}=\left(\frac{2 P_{\mathrm{in}} R}{m \omega_{m}^{2} \omega_{c} Q_{c}}\right)^{1 / 3},
$$

for any value of the input power $P_{\text {in }}$. In contrast, even below the onset of bistability $\left(g x_{s} \ll \gamma\right)$, passive COM systems have very different results

$$
\left|a_{1, s}\right|_{\mathrm{p}}=\left|a_{2, s}\right|_{\mathrm{p}}=\frac{f_{\text {in }}}{\sqrt{2 \gamma}}, \quad x_{s, \mathrm{p}}=\frac{P_{\text {in }}}{2 \gamma m \omega_{m}^{2} R},
$$

where for comparison we take $J \sim \gamma$, and the subscript p denotes the COM system with coupled passive resonators. At the exact gain-loss balance i.e. $\delta \equiv \kappa / \gamma=1$, the ratio of steady-state populations in the passive resonator $\mathbb{R}_{\gamma}$ for $\mathcal{P} \mathcal{T}$-symmetric and passive COM systems is given by

$$
\eta \equiv \frac{\left|a_{2, s}\right|^{2}}{\left|a_{2, s}\right|_{\mathrm{p}}^{2}}=\frac{x_{s}}{x_{s, \mathrm{p}}}=\left(\frac{4 \gamma^{2} m \omega_{m}^{2} R^{2}}{\omega_{c} P_{\mathrm{in}}}\right)^{2 / 3} .
$$

As in relevant experiments [1, 13], the parameter values are taken as $\lambda=1550 \mathrm{~nm}, Q_{c}=3 \times 10^{7}, R \sim 34.5 \mu \mathrm{m}$, and $\omega_{m}=2 \pi \times 23.4 \mathrm{MHZ}, m=5 \times 10^{-11} \mathrm{Kg}, 2 Q_{m} \sim$ $Q_{c} / 10^{5}$, which leads to $g \sim 5.61 \mathrm{GHz} / \mathrm{nm}, \gamma \sim 6.45 \mathrm{MHz}$, $\Gamma_{m}=\omega_{m} / Q_{m} \sim 2.4 \times 10^{5} \mathrm{~Hz}$. For these values, $\Gamma_{m} / \gamma \ll$ 1 , implying that the system is well within the phonon stimulated regime [13]. The condition $g x_{s} \ll \gamma$ is fulfilled for $P_{\text {in }} \ll 137 \mu \mathrm{W}$. 
A main feature of the present work is that, by approaching the gain-loss balance both optical pressure and mechanical gain can be significantly amplified. For $\delta=1$, an enhancement of two orders of magnitude in the intracavity field intensity can be achieved. Namely, $\eta \sim 106$ or $\sim 29.5$ for $P_{\text {in }}=1 \mu \mathrm{W}$ or $7 \mu \mathrm{W}$. The $\mathcal{P} \mathcal{T}$-symmetric COM system performs better than the passive COM system with threshold power $P_{\mathrm{th}, \mathrm{p}}=7 \mu \mathrm{W}$, even at significantly lower input powers $\left(P_{\text {in }} \ll 7 \mu \mathrm{W}\right)[26]$. When the gain-loss ratio deviates from the exact balance condition (e.g., $\delta>3$ ) or when $P_{\text {in }}$ exceeds a threshold $1.1 \mathrm{~mW}$, our system, built from coupled passive and active resonators, behaves in the same way as a system of coupled passive resonators.

Figure 2(a) shows the steady-state populations of intracavity photons in the passive resonator $\mathbb{R}_{\gamma}$. For weak input power $\left(P_{\text {in }} \leq 10 \mu \mathrm{W}\right)$, passive COM systems feature linear responses [1, 5], in sharp contrast to the situation of balanced gain and loss, for which significant optical nonlinearity and the accompanied giant enhancement of COM interactions appear. This is strongly reminiscent of the situation encountered in nonreciprocal wave transmission in $\mathcal{P} \mathcal{T}$-symmetric optical [1] or electric [3] structures. In this nonlinear regime, the transmission rate is vastly different when driving the coupled microresonators from the left or the right side [1, 28]. Similar features as in Fig. 2 can be observed by changing the optical coupling rate $J / \gamma$ at a fixed gain-loss ratio $\kappa / \gamma$ (see the Supplemental Material [26]).

Figure 2(b) shows the associated optical amplification factor $\eta\left(\delta, P_{\mathrm{in}}\right)$, featuring resonance peaks for gain-loss balance. This indicates that for ultraweak input light $\left(P_{\text {in }} \rightarrow 0\right)$, in contrast to the present structure, the intracavity photon number approaches zero very rapidly for passive COM systems. At a fixed value of $P_{\text {in }}$, the radiation pressure in $\mathbb{R}_{\gamma}$ containing the mechanical mode can be significantly enhanced by tuning only the gain-loss ratio in the $\mathcal{P} \mathcal{T}$-symmetric system. This is also reminiscent of resonantly-enhanced light transmissions in $\mathcal{P} \mathcal{T}$ symmetric optical structures [1].

The gain-enhanced nonlinearity is potentially useful in a wide range of phononic engineering systems, e.g. stiffer trapping and further cooling a mechanical object deep into its quantum ground state [1] $[$ ]. Instead of this, here we study its impact on coherent phonon lasing. According to Grudinin et al. [13], compound resonators provide a phonon analog of a two-level laser by replacing the photon-mediated electronic transitions with phononmediated optical transitions. The optical inversion then produces coherent mechanical gain at a breathing mode with frequency $\omega_{m}$, leading to a phonon laser above the threshold power $P_{\mathrm{th}, \mathrm{p}} \sim 7 \mu \mathrm{W}[13]$.

The Hamiltonian of the multi-mode COM system was already given elsewhere [11]. In the rotating frame at the signal laser frequency $\omega_{L}$, the interaction term can
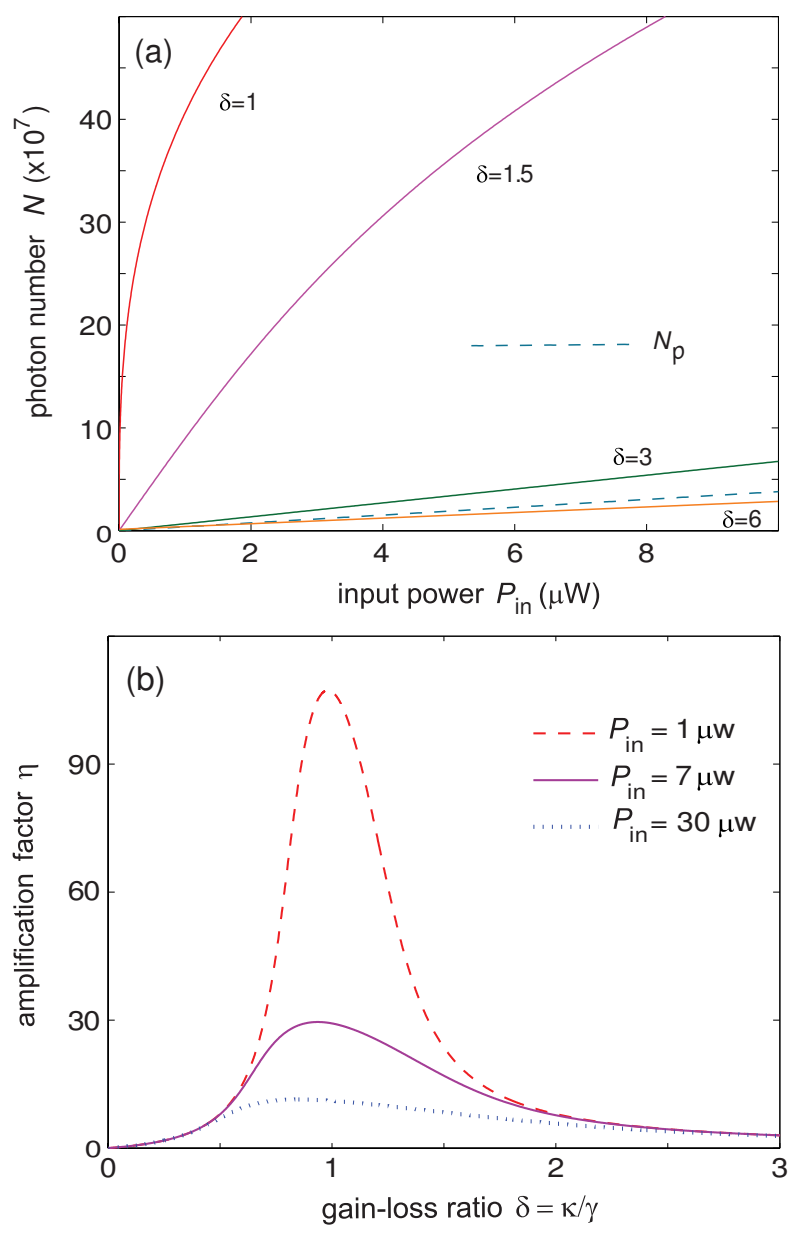

FIG. 2: (Color online) (a) Steady-state cavity-photon number $N$ : for $P_{\text {in }}<30 \mu \mathrm{W}$, the passive system shows linear responses (dashed line); in contrast, nonlinear behavior emerges for the gain-loss balanced system $(\delta=1)$; (b) optomechanical amplification factor $\eta$ with tunable ratio $\delta \equiv \kappa / \gamma$.

be mapped into a simple form, i.e.

$$
H_{\mathrm{int}}=-J\left(a_{1}^{\dagger} a_{2}+h . c .\right)-g a_{2}^{\dagger} a_{2} x \longrightarrow \frac{g x_{0}}{2}\left(p b^{\dagger}+b p^{\dagger}\right),
$$

where the optical inversion operator $p=a_{-}^{\dagger} a_{+}$is defined with the supermode operator $a_{ \pm}=\left(a_{1} \pm a_{2}\right) / \sqrt{2}$. Then we have the equations of motion

$$
\begin{aligned}
& \dot{b}=\left(-\Gamma_{m}-i \omega_{m}\right) b-i \frac{g x_{0}}{2} p, \\
& \dot{p}=(\kappa-\gamma-i \Delta \omega) p-i \frac{g x_{0} \Delta n}{2} b,
\end{aligned}
$$

where $\Delta n=n_{+}-n_{-}, \Delta \omega=\omega_{+}-\omega_{-}$, with $n_{ \pm}$or $\omega_{ \pm}$ being the density or frequency of the supermodes. Here, unlike passive COM systems [11 13], the presence of active gain changes both the mode splitting and linewidth 
of the supermodes, i.e.

$$
\begin{aligned}
& \omega_{ \pm}=\omega_{c} \pm\left[J^{2}-(\kappa+\gamma)^{2} / 4\right]^{1 / 2} \Rightarrow \Delta \omega \neq 0, \\
& \gamma_{ \pm}=(\kappa-\gamma) / 2
\end{aligned}
$$

for strong optical tunnelling rate, i.e. $J \geq(\kappa+\gamma) / 2$, a so-called unbroken- $\mathcal{P} \mathcal{T}$-symmetry regime has been identified experimentally in a purely optical structure [1]. Only in this regime the supermodes can be distributed evenly across the resonators and hence enabling the compensation of loss with gain. In contrast, for the broken$\mathcal{P} \mathcal{T}$-symmetry regime with weaker inter-cavity coupling $J<(\kappa+\gamma) / 2$, no supermode splitting exists at all [26],

$$
\begin{aligned}
& \omega_{ \pm}=\omega_{c} \quad \Rightarrow \Delta \omega=0, \\
& \gamma_{ \pm}=(\kappa-\gamma) / 2 \pm\left[(\kappa+\gamma)^{2} / 4-J^{2}\right]^{1 / 2} .
\end{aligned}
$$

This leads to the following important result: the phonon lasing exists only in the $\mathcal{P} \mathcal{T}$-symmetric regime, where the optical supermodes are non-degenerate and thus can exchange energy through the phonon mode. In contrast, no exchange channel exists at all in the $\mathcal{P} \mathcal{T}$ broken regime where supermodes become spontaneously localized in either the amplifying or the lossy resonator [1]. The presence of optical gain enables one to drive the system between these two regimes on-demand, as well as to controllably set the spectral distance between the supermodes using the interplay between the gain-loss ratio and the intracavity-coupling strength. This is very different from passive COM systems, where the supermode splitting always exists for $J>0$, i.e. $\Delta \omega=2 J[13]$. As a signature of the first-order coherence, the stimulated emission linewidth is much narrower in comparison with that below the lasing threshold [13].

Solving Eq. (10) in the frequency domain gives

$$
p[\omega]=i\left(g x_{0} \Delta n / 2\right) \mathcal{C}[\omega] b,
$$

with a cavity factor

$$
\mathcal{C}[\omega]=[\kappa-\gamma+i(\omega-\Delta \omega)]^{-1} .
$$

Inserting this solution into Eq. (9) gives

$$
\dot{b}=\left[G-\Gamma_{m}-i \omega_{m}-i\left(g x_{0} / 2\right)^{2}(\omega-\Delta \omega)|\mathcal{C}|^{2} \Delta n\right] b,
$$

where the mechanical gain $G$ is given by

$$
G=\frac{\left(g x_{0} / 2\right)^{2} n_{+}(\kappa-\gamma)}{\left(\omega_{+}-\omega_{-}-\omega_{m}\right)^{2}+(\kappa-\gamma)^{2}},
$$

for the blue-supermode threshold density of $n_{+}$at the line center.

The number of emitted phonons is determined by the threshold condition $G=\Gamma_{m}$ (see Fig. 3). This condition, together with $P_{\mathrm{th}}=n_{+}\left(\gamma_{-}+\gamma_{+}\right) \omega_{+}$, yields

$$
P_{\mathrm{th}}=4 \Gamma_{m} \omega_{+}\left[\left(\omega_{+}-\omega_{-}-\omega_{m}\right)^{2}+(\kappa-\gamma)^{2}\right] /\left(g x_{0}\right)^{2} .
$$

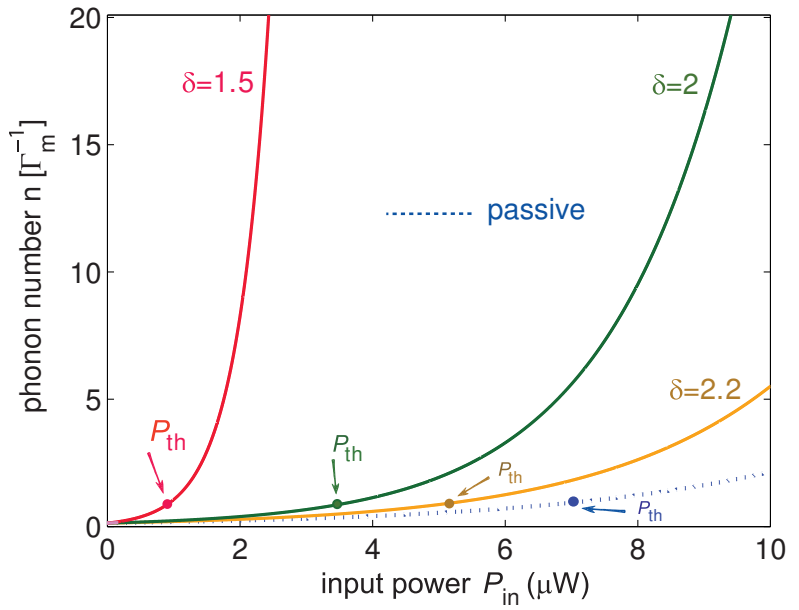

FIG. 3: (Color online) Plot of the stimulated emitted phonon number $n\left[\Gamma_{m}^{-1}\right]=\exp \left[2\left(G-\Gamma_{m}\right) / \Gamma_{m}\right]=\exp \left[2\left(\beta P_{\text {in }} / \omega_{+}-1\right)\right]$ in the $\mathcal{P} \mathcal{T}$-symmetric or the passive system, as a function of $P_{\text {in }}$. Here $\beta \equiv\left(g x_{0}|\mathcal{C}| / 2\right)^{2}$, and to compare with Ref. [13], we choose $2 J=\omega_{m}$ (well within the $\mathcal{P} \mathcal{T}$-symmetric regime). The threshold power $P_{\text {th }}$ denoted by the thick points is obtained for $G=\Gamma_{m}$. The threshold value of the passive COM system is $\sim 7 \mu \mathrm{W}$, agreeing well with the experiment [13], which can be significantly lowered for $\delta \rightarrow 1$.

Clearly $G \rightarrow \infty, P_{\text {th }} \rightarrow 0$ under the following conditions:

$$
\begin{aligned}
& \text { (i) } \omega_{m}=2\left[J^{2}-(\kappa+\gamma)^{2} / 4\right]^{1 / 2}, \\
& \text { (ii) } \kappa=\gamma \text { (gain-loss balance), }
\end{aligned}
$$

indicating an ultralow-threshold phonon laser by tuning both $\kappa / \gamma$ and $J / \gamma$ (in the $\mathcal{P} \mathcal{T}$-symmetric regime), which is otherwise unattainable for passive COM devices. The tunability of these parameters was already demonstrated in a very recent experiment [1]. With the parameter values given above, the threshold power of the passive COM system can be estimated as $P_{c T, \mathrm{p}} \sim 7 \mu \mathrm{W}$, agreeing well with the experiment [13]. In contrast, by compensating loss with gain $(\delta \rightarrow 1)$, the phonon-lasing threshold can be significantly lowered, also in agreement with the resonant enhancement shown in Fig. 2. We see that the two tunable parameters (the optical tunnelling rate and the gain-loss ratio), when acting in concert, provide more flexibility in COM control.

Finally, note that the presence of optical gain provides an additional degree of freedom to control the dynamics of the COM system. Namely, tuning the gain-loss ratio can switch between stable and bistable operations (see the Supplemental Materials [26]). A similar situation can also occur in electronic circuits [3].

In summary, we have studied a compound-resonator COM system in the presence of active gain in one resonator and passive loss in the other. At the gain-loss balance, an optical nonlinearity is observable for steadystate populations even for ultraweak input powers. This 
gain-induced nonlinearity leads to a giant enhancement of both optical pressure and mechanical gain, enabling then an ultralow-threshold phonon laser in the $\mathcal{P} \mathcal{T}$-symmetric regime. As demonstrated by a recent experiment [13], increasing the optical tunnelling rate can lead to a transition from broken to unbroken $\mathcal{P} \mathcal{T}$-symmetric regimes, i.e. realizing not only the exchange of two subsystems but also changing gain to loss and vice versa. The linearto-nonlinear transition, i.e. the giant enhancement of the intracavity field intensity and then the mechanical gain, can be realized when approaching the gain-loss balance. We stress that $\kappa / \gamma \rightarrow 1$ and $J \geq(\kappa+\gamma) / 2$ should be satisfied simultaneously to observe the unidirectional propagation of light in a purely optical experiment [1], and now to obtain an ultralow-threshold phonon laser in a COM system.

Our work opens up exciting new perspectives for $\mathrm{COM}$ control with unconventional $\mathcal{P} \mathcal{T}$-structures 17 19], e.g., asymmetric wave transport [3], gain-enhanced mechanical mixing [24], ultraslow light in $\mathcal{P} \mathcal{T}$-structures [12, 29], and $\mathcal{P} \mathcal{T}$-enhanced phononic squeezing or entanglement [6]. In future works we also plan to study a CW phonon laser with a three-level system [14], a multi-mode phonon laser with a tunable gain-loss ratio, or a triple-resonator set-up [24, 30] with two active elements on both sides of a mechanical mode. In view of rapid advances in compound micro-structures [1, 11 -13, 21], particularly those on phonon lasers 13] and $\mathcal{P} \mathcal{T}$-symmetric resonators [1], we believe that these studies will be highly accessible in experiments in the near future.

HJ thanks Y. Nakamura, K. Usami, and R. Yamazaki for helpful discussions. HJ is supported by the NSFC under Grant No.11274098 and the Henan Excellence Plan (T555-1202). SKO and LY are supported by ARO Grant No. W911NF-12-1-0026 and the NSFC under Grant No. 61328502. XYL is supported by NSFC under Grant No. 11374116. JZ is supported by the NSFC under Grant Nos. 61174084, 61134008, and the NBRPC (973 Program) under Grant No. 2014CB921401. FN is supported by the RIKEN iTHES Project, MURI Center for Dynamic Magneto-Optics, and a Grant-in-Aid for Scientific Research (S).

[1] M. Aspelmeyer, P. Meystre and K. Schwab, Physics Today 65, 29 (2012); T. J. Kippenberg and K. J. Vahala, Science 321, 1172 (2008); M. Aspelmeyer, T. J. Kippenberg, and F. Marquardt, arXiv:1303.0733 (2013).

[2] Y. S. Park and H. Wang, Nature Phys. 5, 489 (2009); A. Schliesser, et al., ibid. 5, 509 (2009).

[3] A. D. O'Connell, et al., Nature (London) 464, 697 (2010); J. D. Teufel, et al., ibid. 475, 359 (2011); J. Chan, et al., ibid. 478, 89 (2011).

[4] T. P. Purdy, R. W. Peterson, and C. A. Regal, Science 339, 801 (2013).
[5] E. Verhagen, et al., Nature (London) 482, 63 (2012); J. Teufel, et al., ibid. 471, 204 (2011).

[6] D. W. C. Brooks, et al., Nature (London) 488, 476 (2012); A. H. Safavi-Naeini, et al., ibid. 500, 185 (2013); T. A. Palomaki, et al. Science 342, 710 (2013).

[7] I. Pikovski, et al., Nature Phys. 8, 393 (2012).

[8] J. Gieseler, L. Novotny, and R. R. Quidant, Nature Phys. 9, 806 (2013).

[9] C. Dong, et al. Science 338, 1609 (2012); J. T. Hill, et al. Nature Commun. 3, 1196 (2012).

[10] T. A. Palomaki, et al. Nature (London) 495, 210 (2013); J. Bochmann, et al. Nature Phys. 9, 712 (2013).

[11] K. Stannigel, et al., Phys. Rev. Lett. 109, 013603 (2012); M. Ludwig, et al., ibid. 109, 063601 (2012); P. Komar, et al., Phys. Rev. A 87, 013839 (2013).

[12] B. Peng, et al., arXiv:1404.5941 (2014); B. Peng, et al., Opt. Lett. 37, 3435 (2012); J. Fan and L. Zhu, Opt. Express 20, 20790 (2012).

[13] I. S. Grudinin, et al., Phys. Rev. Lett. 104, 083901 (2010).

[14] K. Vahala, et al., Nature Phys. 5, 682 (2009); J. B. Khurgin, et al., Phys. Rev. Lett. 108, 223904 (2012); I. Mahboob, et al., ibid. 110, 127202 (2013); U. Kemiktarak, et al. arXiv:1402.0714 (2014).

[15] D. Hatanaka, et al., Appl. Phys. Lett. 102, 213102 (2013); M. D. LaHaye, et al., Nature (London) 459, 960 (2009); C. Genes, et al., Phys. Rev. A 78, 032316 (2008).

[16] N. Li, et al., Rev. Mod. Phys. 84, 1045 (2012).

[17] C. M. Bender and S. Boettcher, Phys. Rev. Lett. 80, 5243 (1998); C. M. Bender, Rep. Prog. Phys. 70, 947 (2007); C. M. Bender, et al., Phys. Rev. A 88, 062111 (2013).

[18] A. Regensburger, et al., Nature (London) 488, 167 (2012); C. E. Rüter, et al., Nature Phys. 6, 192 (2010); A. Guo, et al., Phys. Rev. Lett. 103, 093902 (2009).

[19] G. S. Agarwal and K. Qu, Phys. Rev. A 85, 031802(R) (2012).

[20] B. Peng, et al., Nature Phys. 10, 394 (2014).

[21] F. Monifi, S. K. Özdemir, and L. Yang, Appl. Phys. 103, 181103 (2013); M. Zhang, et al., Phys. Rev. Lett. 109, 233906 (2012); L. He, et al., IEEE J. Quant. Elect. 46, 1626 (2010).

[22] S. Mancini and P. Tombesi, Phys. Rev. A 49, 4055 (1994).

[23] A. Nunnenkamp, K. Borkje, and S. M. Girvin, Phys. Rev. Lett. 107, 063602 (2011); P. Rabl, ibid. 107, 063601 (2011).

[24] Q. Lin, et al., Nature Photon. 4, 236 (2010); H. Okamoto, et al., Nature Phys. 9, 480 (2013); A. B. Shkarin, et al., Phys. Rev. Lett. 112, 013602 (2014).

[25] S. Aldana, C. Bruder, and A. Nunnenkamp, Phys. Rev. A 88, 043826 (2013).

[26] For more details, see Supplemental Materials.

[27] N. Bender, et al., Phys. Rev. Lett. 110, 234101 (2013).

[28] L. Fan, et al., Science 335, 447 (2012); H. Lira, et al. Phys. Rev. Lett. 109, 033901 (2012).

[29] G. S. Agarwal and S. Huang, Phys. Rev. A 81, 041803(R) (2010).

[30] C. Schmidt, et al., Appl. Phys. Lett. 103, 041115 (2013). 


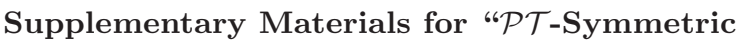 Phonon Laser"}

This document consists of three parts: (I) amplification factor with varying the optical tunnelling rate $J$; (II) supermode splitting and linewidth; and (III) stability analysis with the tunable gain-loss ratio $\delta$.

\section{Amplification factor with varying $J$}

Figure 2 in the main text shows the steady-state populations of intracavity photons in the passive resonator, by numerically solving Eq. (5). A notable feature of the $\mathcal{P} \mathcal{T}$ symmetric COM system is the emerging resonance peaks of the optical amplification factor $\eta$ around the gain-loss balance $\delta=1$ (see Fig. 2b), where we choose to change the values of gain-loss ratio $\delta$ but with fixed optical tunnelling rate $(J / \gamma=1)$. Here we show in Fig. S1 that for fixed $\delta$, similar features can be observed by changing the optical tunnelling rate $J / \gamma$.

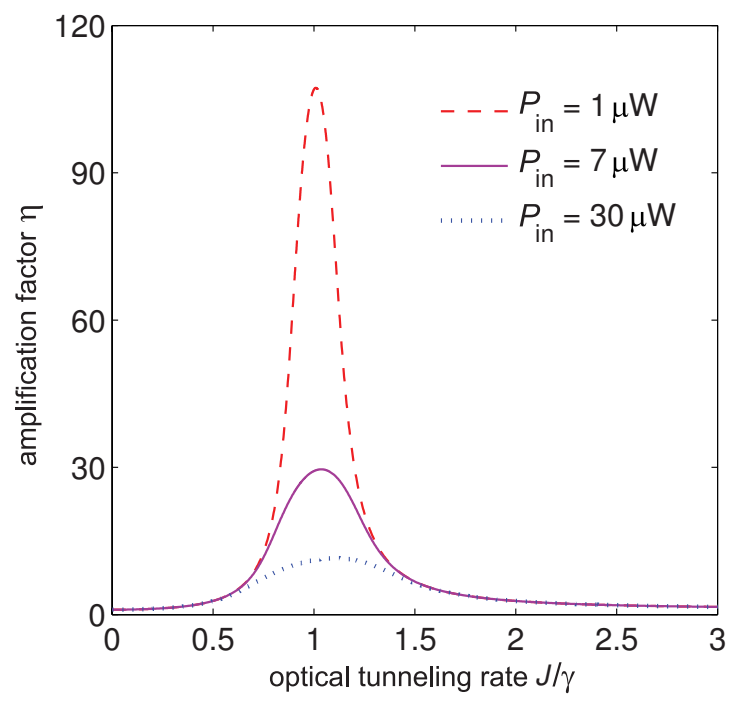

FIG. 4: (Color online) Optomechanical amplification factor $\eta$ versus the tunable optical tunnelling rate $J / \gamma$, for the fixed value of gain-loss ratio $\delta=1$. Here the amplification factor $\eta$ is by the Eq. (7) of the main text (having the $\mathcal{P} \mathcal{T}$-symmetric result divided by the result for the passive COM case).

Even when comparing with a passive COM system with threshold power $P_{\mathrm{th}, \mathrm{p}}=7 \mu \mathrm{W}$, the $\mathcal{P} \mathcal{T}$-symmetric COM system performs better for significantly lower input power, i.e.

$$
\xi \equiv \frac{x_{s}\left(\delta, P_{\mathrm{in}}\right)}{x_{s, \mathrm{p}}\left(P_{\mathrm{in}}=P_{\mathrm{th}, \mathrm{p}}\right)} \geq 1
$$

which, for $\delta=1$, can be realized for $P_{\text {in }} \geq 3 \times 10^{-4} \mu \mathrm{W}$. For instance, $\xi \sim 15.9$ or 29.5 for $P_{\text {in }}=1 \mu \mathrm{W}$ or

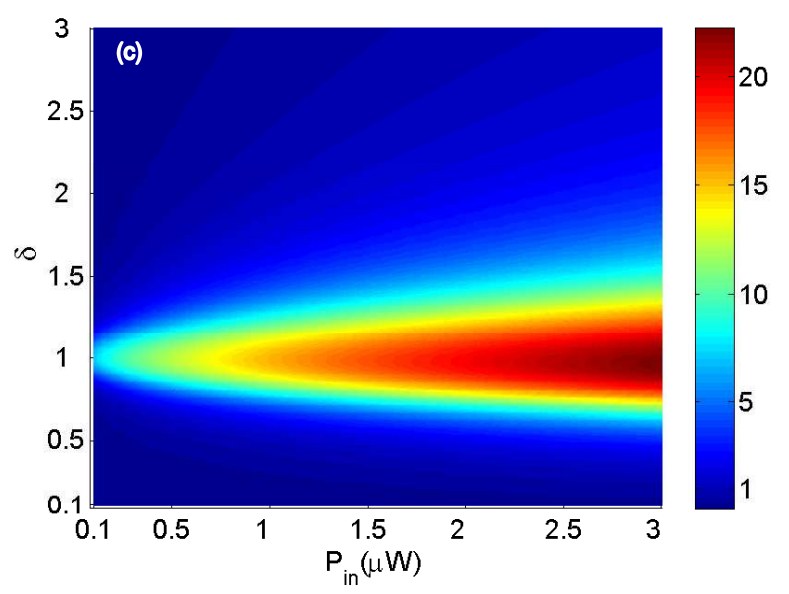

FIG. 5: (Color online) Relative amplification factor $\xi$ versus tunable gain-loss ratio $\delta \equiv \kappa / \gamma$ and the input power $P_{\text {in }}$. The vertical color bar refers to the values of $\xi$.

$7 \mu \mathrm{W}$. Figure $\mathrm{S} 2$ plots $\xi\left(\delta, P_{i n}\right)$, by comparing the $\mathcal{P} \mathcal{T}$ symmetric system working below the threshold $7 \mu \mathrm{W}$ and the passive COM system working with $7 \mu \mathrm{W}$. For $\delta \rightarrow 1$, $P_{\text {in }}>0.1 \mu \mathrm{W}$, the enhancement effect is significant even in this situation.

\section{Supermode splitting and linewidth}

The non-Hermitian Hamiltonian of the system (comprising the optical gain, the optical supermodes, and the phonon mode) can be written at the simplest level as

$$
\begin{aligned}
H_{\mathrm{tot}}= & \left(\omega_{+}-i \gamma_{+}\right) a_{+}^{\dagger} a_{+}+\left(\omega_{-}-i \gamma_{-}\right) a_{-}^{\dagger} a_{-} \\
& +\omega_{m} b^{\dagger} b+\frac{g x_{0}}{2}\left(b a_{+}^{\dagger} a_{-}+a_{-}^{\dagger} a_{+} b^{\dagger}\right) .
\end{aligned}
$$

The weak driving terms are not explicitly shown here. The specific expressions of $\omega_{ \pm}$and $\gamma_{ \pm}$are different in two distinct regimes (see the main text): (i) the regime which was identified as the broken- $\mathcal{P} \mathcal{T}$-symmetry phase for a purely optical structure [1], characterized by $(\kappa+\gamma) / 2>J$; (ii) the regime with strong inter-cavity coupling $(\kappa+\gamma) / 2 \leq J$, which for a purely optical system, was identified experimentally as the unbroken- $\mathcal{P} \mathcal{T}$ symmetry phase [1]. We note that only the supermodes with unbroken $\mathcal{P} \mathcal{T}$-symmetry can be distributed evenly across the coupled resonators, hence enabling the compensation of loss with gain. In contrast to this, the $\mathcal{P} \mathcal{T}$ broken supermodes become spontaneously localized in either the amplifying or lossy resonator, hence experiencing either net gain or loss (see Ref. 2] for more details).

As Fig. S3 shows, the phonon lasing action can exist only in the $\mathcal{P} \mathcal{T}$-symmetric regime where the optical supermodes are non-degenerate and thus can exchange energy through the phonon mode. Figure S3 is similar to 

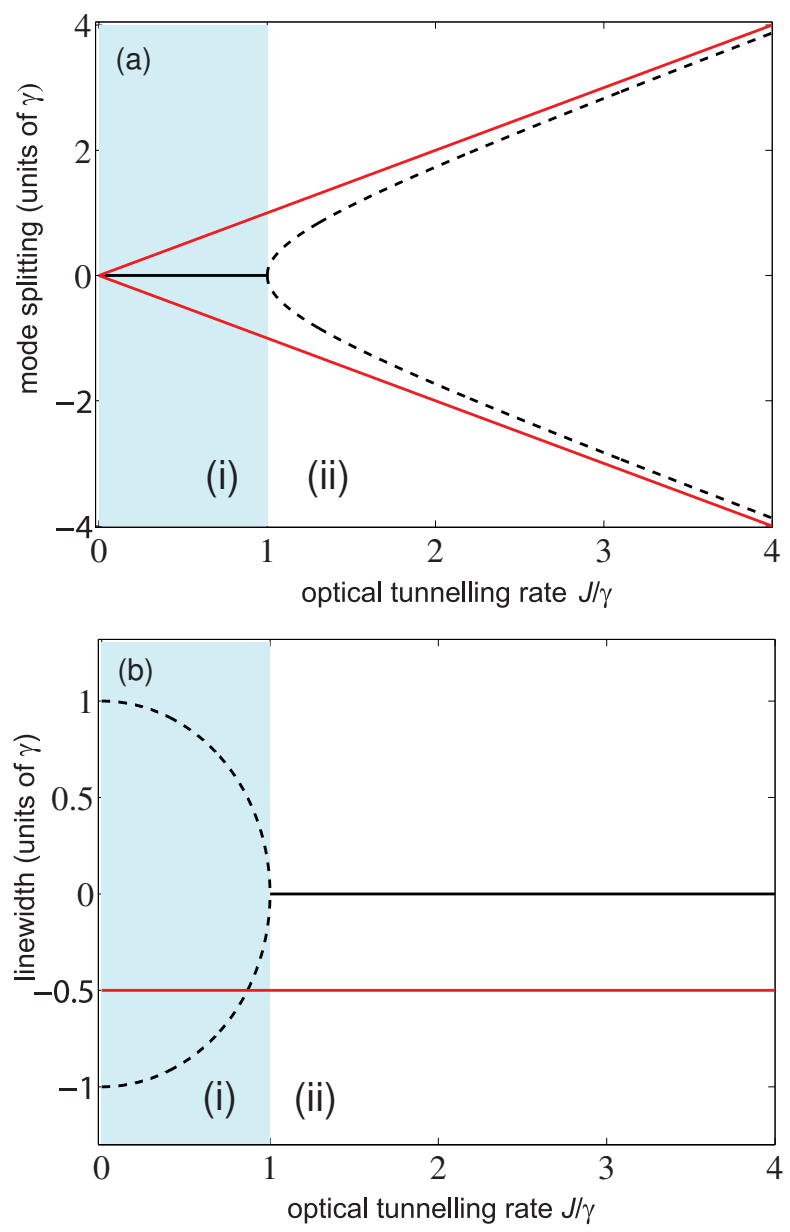

FIG. 6: (Color online) Mode-splitting (a) and linewidth (b) of the supermodes in the $\mathcal{P} \mathcal{T}$-symmetric COM system (black curves) or the passive COM system (red lines), as a function of $J / \gamma$ (see also Ref. [2]). The PT-symmetry holds in (ii) and not in (i).

that as was shown in all $\mathcal{P} \mathcal{T}$-symmetric systems, e.g. in Ref. [1]; nevertheless, here we show for the first time that the ultralow-threshold phonon lasing can exist only in the unbroken-symmetry regime, not in the broken-symmetry regime.

We stress that, by increasing the optical coupling rate $J$, one can realize the transition from the broken to the unbroken $\mathcal{P} \mathcal{T}$-symmetric regimes. That is, realizing not only the exchange of two subsystems (micro-resonators), but also changing the gain to loss and vice versa. By tuning the gain-loss ratio, one can realize the transition from linear to nonlinear regimes, i.e. the giant enhancement of the intracavity field intensity and then the mechanical gain. Both of these two conditions (strong $J$ and $\delta=1$ ) are required to observe the unidirectional wave propagation in a purely optical system [1] and now the ultralow-threshold phonon laser in a COM system. It is the presence of active gain which makes it possible to realize these two conditions simultaneously.

\section{Stability analysis with tunable $\delta$}

Finally, we mention that in the vicinity of the gainloss balance, the stability properties of the COM system can also be significantly modified. To see this we need to study the role of thermal noise on the mechanical response. This is accomplished by linearizing Eqs. (13 ) and then studying the fluctuations of the operators. With the equations of motion as Eqs. (1-3) in the main text, including also the optical detunings $\Delta_{i}=\omega_{c, i}-\omega_{L}$ $(i=1,2)$ between the two resonators and the input signal laser, the mean values of the optical and mechanical modes then satisfy the following equation

$$
\begin{aligned}
\left(\kappa-i \Delta_{1}\right) a_{1, s}-i J a_{2, s}-\sqrt{2 \kappa} \Omega_{d} & =0 \\
\left(-\gamma-i \Delta_{2}\right) a_{2, s}-i g a_{2, s} x-i J a_{1, s} & =0 \\
-m \omega_{m}^{2} x_{s}-g\left|a_{2, s}\right|^{2} & =0
\end{aligned}
$$

with $\Omega_{d}=\sqrt{P_{\text {in }} / \hbar \omega_{c}}$. From these equations we can obtain the following polynomial for the input power $P_{\text {in }}$,

$$
P_{\text {in }}=\frac{\omega_{c, 1}}{2 \kappa J^{2}}\left[\begin{array}{c}
\frac{g^{4}}{m^{2} \omega_{m}^{4}}\left(\Delta_{1}^{2}+\kappa^{2}\right) N^{3}+\frac{g^{2}}{m \omega_{m}^{2}}\left(2 \gamma \Delta_{1} \kappa+2 J^{2} \Delta_{1}-\Delta_{1}^{2} \Delta_{2}-2 \gamma \kappa \Delta_{1}-2 \kappa^{2} \Delta_{2}\right) N^{2} \\
+\left(\gamma^{2} \kappa^{2}+J^{4}-2 \gamma \kappa J^{2}+\Delta_{1}^{2} \Delta_{2}^{2}+2 \gamma \kappa \Delta_{1} \Delta_{2}-2 J^{2} \Delta_{1} \Delta_{2}+\gamma^{2} \Delta_{1}^{2}+\kappa^{2} \Delta_{2}^{2}-2 \gamma \Delta_{1} \kappa \Delta_{2}\right) N
\end{array}\right],
$$

where $N$ denotes the photon number inside the passive resonator. For a passive COM system, increasing the input power can lead to unstable evolutions of the system. For our present active system, however, a bistability-free regime is accessible by tuning $\delta$, which is reminiscent of that in a $\mathcal{P} \mathcal{T}$-symmetric electronics system [3] .

Now by applying the expansions: $a_{1}=a_{1, s}+\delta a_{1}, a_{2}=$ $a_{2, s}+\delta a_{2}, \quad x=x_{s}+\delta x$, it is straightforward to linearize
Eqs. (1-3) to obtain

$$
\begin{aligned}
& \frac{d \delta a_{1}}{d t}=\kappa \delta a_{1}-i J \delta a_{2}+\sqrt{2 \kappa} \delta a^{\text {in }}, \\
& \frac{d \delta a_{2}}{d t}=-\gamma \delta a_{2}-i J \delta a_{1}-i g a_{2, s} \delta x-i g x_{s} \delta a_{2}, \\
& \frac{d^{2} \delta x}{d t^{2}}+\Gamma_{m} \frac{d \delta x}{d t}+\omega_{m}^{2} \delta x=\frac{g}{m}\left(a_{2, s}^{*} \delta a_{2}+a_{2, s} \delta a_{2}^{*}\right)+\frac{\delta \varepsilon^{\text {in }}}{m},
\end{aligned}
$$




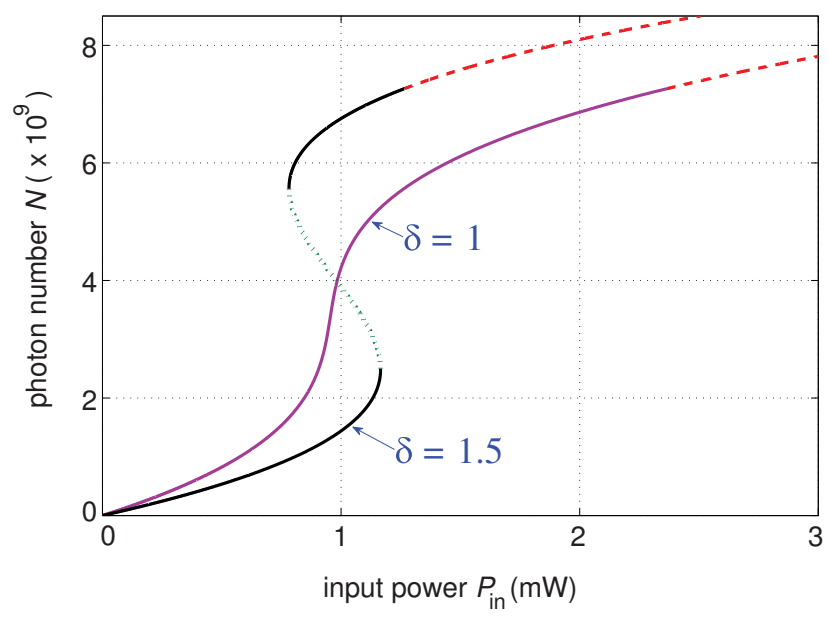

FIG. 7: (Color online) Mean population of photons in the passive resonator containing the mechanical mode. The bistability feature seen for $\delta=1.5$, which is confirmed to be similar to the passive COM situation, can be removed at the gainloss balance. Dotted and dashed curves denote different types of instability (see Ref. [5]). All relevant parameter values are given in the main text, including here also the optical detunings $\Delta_{1} / \omega_{m}=0.03$ and $\Delta_{2} / \omega_{m}=0.15$. for these fluctuations. The resulting solutions can be compactly written as

$$
\delta x[\omega]=\chi[\omega] \delta \varepsilon^{\text {in }}
$$

where the susceptibility is in the form

$$
\chi^{-1}[\omega]=m\left(\omega_{m}^{2}-\omega^{2}-i \omega \Gamma_{m}+\frac{2 g^{2}}{m}\left|a_{2, s}\right|^{2} \mathbf{R e} \mathbb{Y}[\omega]\right),
$$

with a cavity factor

$$
\mathbb{Y}^{-1}[\omega]=-\omega-i \gamma+g x_{s}+\frac{J^{2}}{\omega-i \kappa}
$$

for a thermally-driven system. Hence, by tuning the gainloss ratio, COM properties (such as the mechanical susceptibility [4] and the bistability features) can be significantly modified. As a specific example, Fig. S4 shows the stable and unstable parameter regimes, by applying the Routh-Hurwitz criterion [5].

By applying this criterion to the coefficient matrix of these linear equations, we obtain in the following two stability conditions

$$
\begin{aligned}
& S_{1}=\left(\omega_{m}^{2}+\frac{\Gamma_{m}^{2}}{4}\right)\left(\triangle_{2}^{2}+\frac{\gamma^{2}}{4}\right)-4 \omega_{m} G^{2} \triangle_{2}>0 \\
& S_{2}=\gamma \Gamma_{m}\left[\left(\triangle_{2}^{2}-\omega_{m}^{2}\right)^{2}+\frac{1}{2}\left(\triangle_{2}^{2}+\omega_{m}^{2}\right)\left(\gamma+\Gamma_{m}\right)^{2}+\frac{1}{16}\left(\gamma+\Gamma_{m}\right)^{4}\right]+4 G^{2} \triangle_{2} \omega_{m}\left(\gamma+\Gamma_{m}\right)^{2}>0
\end{aligned}
$$

where $G=g x_{0}\left|a_{2, s}\right|$. For $\triangle_{2} \geq 0$, the second inequality is always satisfied. With the help of these conditions, the bistability lines can then be plotted by numerically evaluating the polynomial in Eq. (S5). The resulting figure is shown in Fig. S4, with the corresponding stable regimes of parameters. Here, to compare with passive COM systems, we include also optical detunings, ignored previously in order to focus on the role of optical gain. We see that, in general, for stronger input power, bistability appears for higher gain-loss imbalance, as in passive COM systems. In contrast, this effect can now be completely removed at the gain-loss balance (see also Ref. [3] ).

[1] B. Peng, et al., Nature Phys. 10, 394 (2014).

[2] C. M. Bender, et al., Phys. Rev. A 88, 062111 (2013).

[3] N. Bender, et al., Phys. Rev. Lett. 110, 234101 (2013).

[4] S. Mancini and P. Tombesi, Phys. Rev. A 49, 4055 (1994).

[5] S. Aldana, C. Bruder, and A. Nunnenkamp, Phys. Rev. A 88, 043826 (2013). 